\title{
Modélisation du manteau neigeux polaire
}

\author{
par H. Dang, Christophe Genthon \\ LGGE, CNRS (Université Joseph Fourier) \\ E. Martin \\ Météo France, Centre national de recherche météorologique
}

\section{Introduction}

CROCUS [2,3] est un modèle unidimensionnel simulant l'évolution du manteau neigeux au cours du temps en fonction des conditions météorologiques au dessus de la surface de neige. Il a besoin de 9 paramètres météorologiques au pas horaire qui sont :

- la température de l'air,

- l'humidité relative de l'air,

- la vitesse du vent,

- le rayonnement solaire incident direct et diffus,

- le rayonnement infrarouge incident,

- la nébulosité

- la quantité de précipitation et son type.

En régions polaires, l'observation de ces paramètres n'est disponible qu'en un nombre très limité de sites et sur des durées courtes. Il n'est donc pas possible de développer une modélisation du manteau neigeux à la surface des calottes de glace polaire à partir des seules observations. Par contre plus de 15 années d'analyses et de prévisions météorologiques sont archivées au Centre Européen de Prévisions Météorologiques à Moyen Terme (CEPMMT, Reading. GB) et sont disponibles à une fréquence de 6 heures et une résolution spatiale atteignant $0.5^{\circ}$ sur l'ensemble du globe. Ces analyses et prévisions sont contraintes par les observations synoptiques réalisées et transmises par le réseau météorologique mondial, mais puisqu'en régions polaires ces observations sont rares la contrainte est faible. De plus, le rayonnement et les précipitations ne sont pas systématiquement observés et transmis par le réseau. Au dessus des calottes de glace polaires, l'archive du CEPMMT est donc largement un produit de modèle climatique, dont il convient de soigneusement vérifier la qualité et l'impact d'éventuels défauts sur les résultats de CROCUS.

CROCUS a été développé et validé pour la simulation de neiges tempérées alpines. On s'attend à ce que, dans les conditions de froid intense, de précipitations faibles et de cycle radiatif diurne peu marqué caractéristiques des régions polaires, certaines des paramétrisations mises en oeuvre atteignent leur limite de validité. Pour vérifier cet aspect, un site extrême mais pour lequel de bonnes données d'observation de la neige et du forçage météorologique sont disponibles doit être choisi. La station permanente du Pole Sud en Antarctique constitue probablement le meilleur site de test possible.

\section{Extraction des données météorologiques des archives du CEPMMT et validation en Antarctique}

Les analyses du CEPMMT ont été extraites pour toute la surface antarctique sur une grille de $1^{\circ} 5$ de résolution avec une fréquence de 6 heures et, dans un premier temps, sur une période de un an seulement allant du lor octobre 1991 au 30 septembre 1992. Les observations de stations météorologiques automatiques (Automatic Weather Stations, AWS) aux quatre sites antarctiques de Pole Sud, Lettau (sur le shelf de Ross), Byrd (en Antarctique de l'ouest), et Dôme C (en Antarctique de l'est) ont été également compilées pour la même période, afin de comparer les analyses à des observations réelles et simultanées. Les AWS mesurent toutes les 10 minutes la pression, la température, la direction et la force du vent à deux mètres. Enfin, quelques observations publiées de rayonnement à Pole Sud seront aussi comparées aux données du CEPMMT.

Les valeurs météorologiques au pas horaire pour forcer CROCUS sont obtenues par interpolation temporelle. Certaines variables nécessaires à CROCUS ne sont pas directement récupérables de l'archive CEPMMT. Ainsi, les précipitations et les rayonnements extraits de l'archive sont des flux prévus cumulés pendant un jour de simulation. De plus, les rayonnements visible et infrarouge sont des bilans nets alors que CROCUS nécessite les composantes de flux direct et diffus incidents dans le visible, et incident et réémis dans l'infrarouge. La partie réfléchie du visible est recalculée à partir de l'albédo, ce qui permet de déduire le visible incident total. La nébulosité issue de l'archive permet alors d'estimer la fraction diffuse et donc aussi la partie directe. L'émission infrarouge de la surface est simplement calculée à partir de la température de surface.

Après cette décomposition, il reste à reconstruire le cycle diurne du rayonnement visible afin d'évaluer les valeurs instantanées à chaque heure de la journée à partir des données cumulées. Pour cela, l'insolation théorique est recalculée grâce aux formules astronomiques, et l'on redistribue la valeur cumulée suivant le cycle théorique.

La Figure I présente les différentes composantes au pas horaire du bilan radiatif obtenues à partir de l'archive CEPMMT. Ces données sont comparées aux rares observations publiées, rapportées par Carroll [4] et correspondant aux années 1976 et 1977. En bilan net intégré sur une année, les données CEPMMT excèdent les observations de 14.79 $\mathrm{Wm}^{-2}$ dans le domaine visible et les sous-estiment de $9.6 \mathrm{Wm}^{-2}$ dans le domaine infra-rouge. L'albédo visible de l'archive CEPMMT est d'environ $80 \%$, légèrement en deçà des valeurs observées, ce qui peut expliquer l'excédent d'énergie solaire absorbée. Dans l'infrarouge, c'est la partie incidente du rayonnement qui, pour une raison encore inconnue, est trop élevée d'environ $10 \mathrm{Wm}^{-2}$.

La température de l'air à $2 \mathrm{~m}$ (fig. 2), montre que les analyses sont trop froides de $3.3^{\circ} \mathrm{C}$ en moyenne annuelle. Si l'on sépare les saisons été-hiver, on s'aperçoit que la température estivale est bien reproduite, et que c'est la saison d'hiver qui est en moyenne trop froide. L'amplitude des réchauffements rapides que l'on observe pendant la saison froide sont mal 


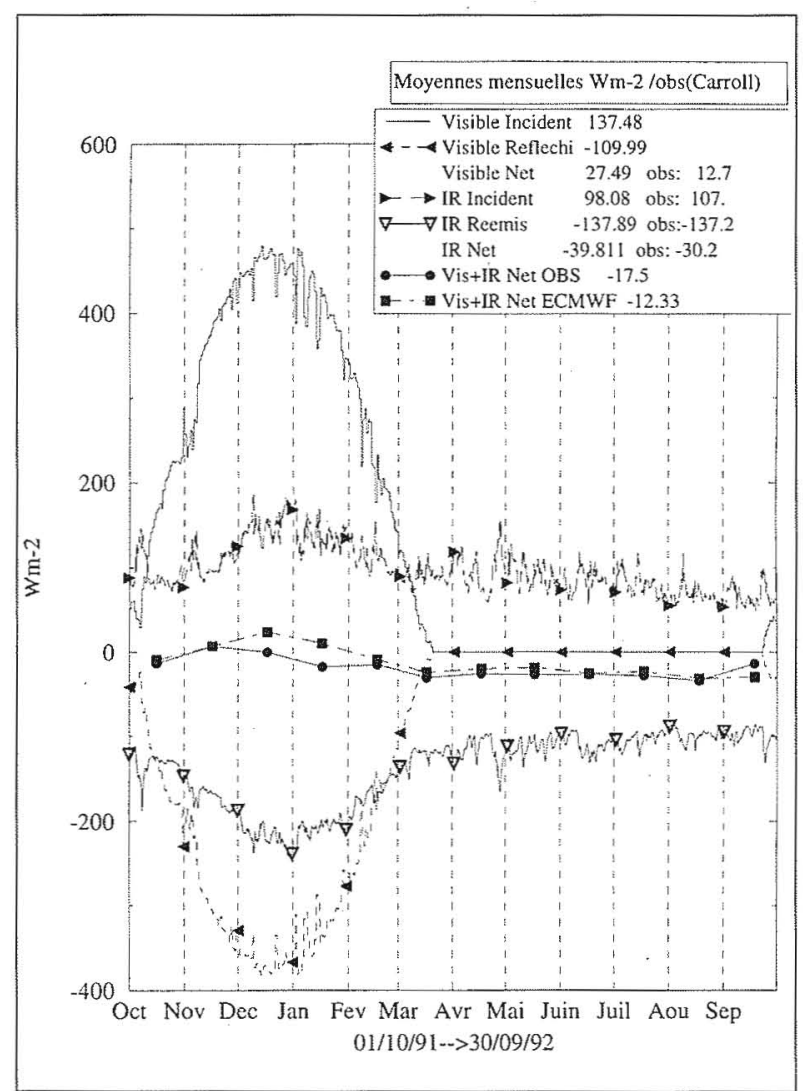

1. Bilan radiatif à Pole Sud

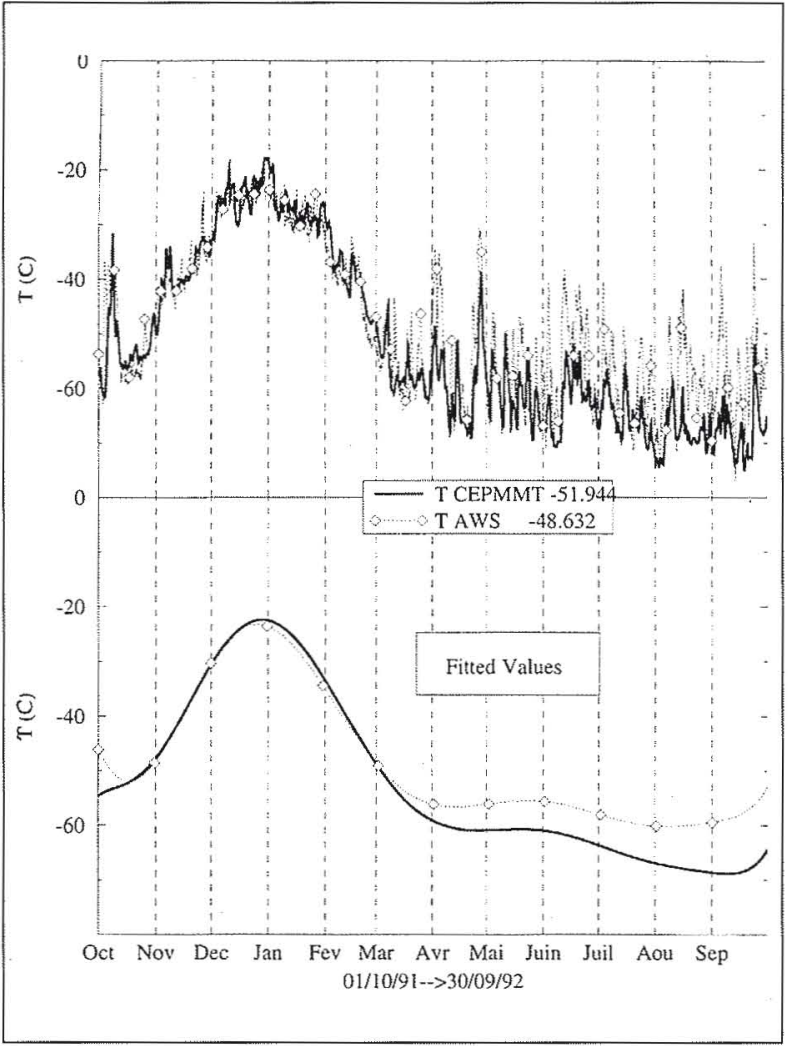

2. Température de l'air à Pole Sud reproduits dans l'archive CEPMMT, et il en résulte des erreurs de courte durée mais répétées pouvant atteindre $20^{\circ} \mathrm{C}$.

La vitesse du vent (fig. 3) est en moyenne annuelle bien représentée $\left(5.35 \mathrm{~ms}^{-1}\right.$ pour les analyses et $5.44 \mathrm{~ms}^{-1}$ pour les AWS). On note cependant un léger déphasage dans les épisodes de vent, qui s'accentue pour les mois d'hiver.

Bien que l'archive CEPMMT constitue une référence en climatologie et une source de données météorologiques unique, en particulier pour notre application, nous avons montré qu'elle présente en Antarctique des imprécisions. La sensibilité du modèle de neige aux données météorologiques en entrée doit donc être évaluée afin d'estimer la qualité potentielle des futures simulations du manteau neigeux à grande échelle.

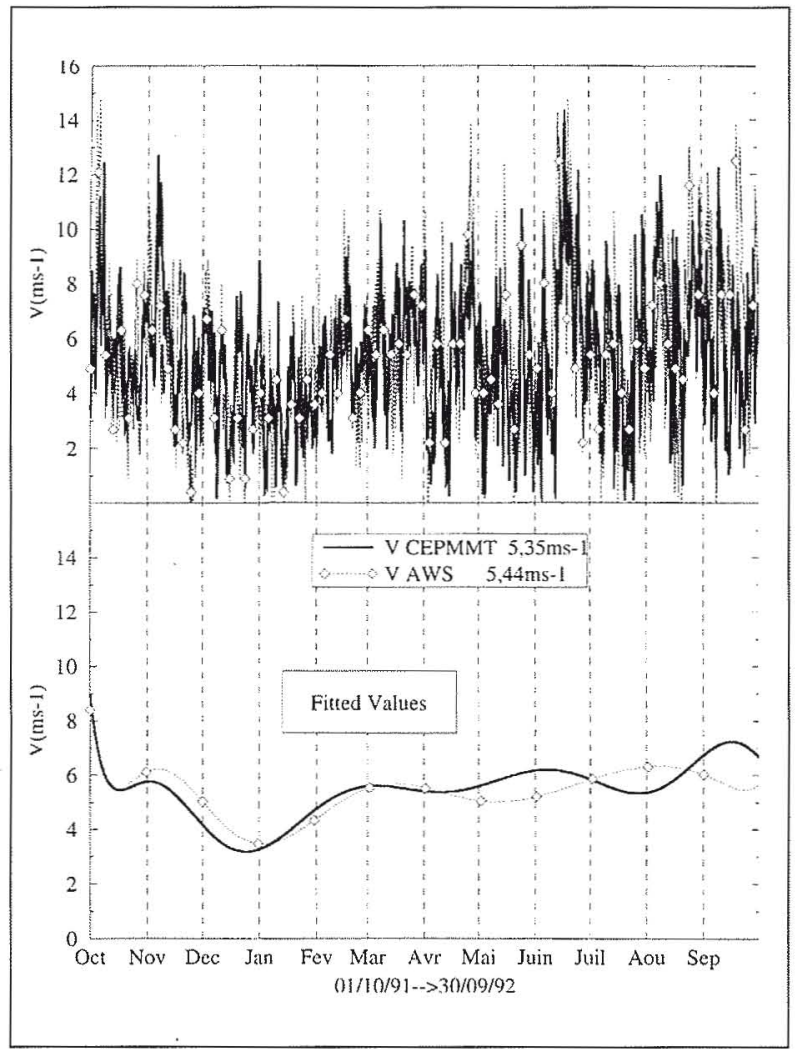

3. Vitesse du vent à Pole Sud

\section{Résultats de simulations à Pole Sud}

Dans ce chapitre sont présentés les résultats de tests successifs de la sensibilité de CROCUS à la donnée de température de surface, à celle du rayonnement infra-rouge incident, à la densité de surface de la neige fraîche, et à la variation interannuelle de l'accumulation. Pour ces tests, le modèle de neige est intégré pendant 20 ans en utilisant de façon cyclique d'une année sur l'autre les données météorologiques décrites ci-dessus. La variation interannuelle de l'accumulation sera introduite en perturbant d'une année sur l'autre la donnée météorologique.

\subsection{Température}

La figure 4 représente les profils de température moyenne mensuelle à l'intérieur du manteau neigeux jusqu'à 2 mètres 


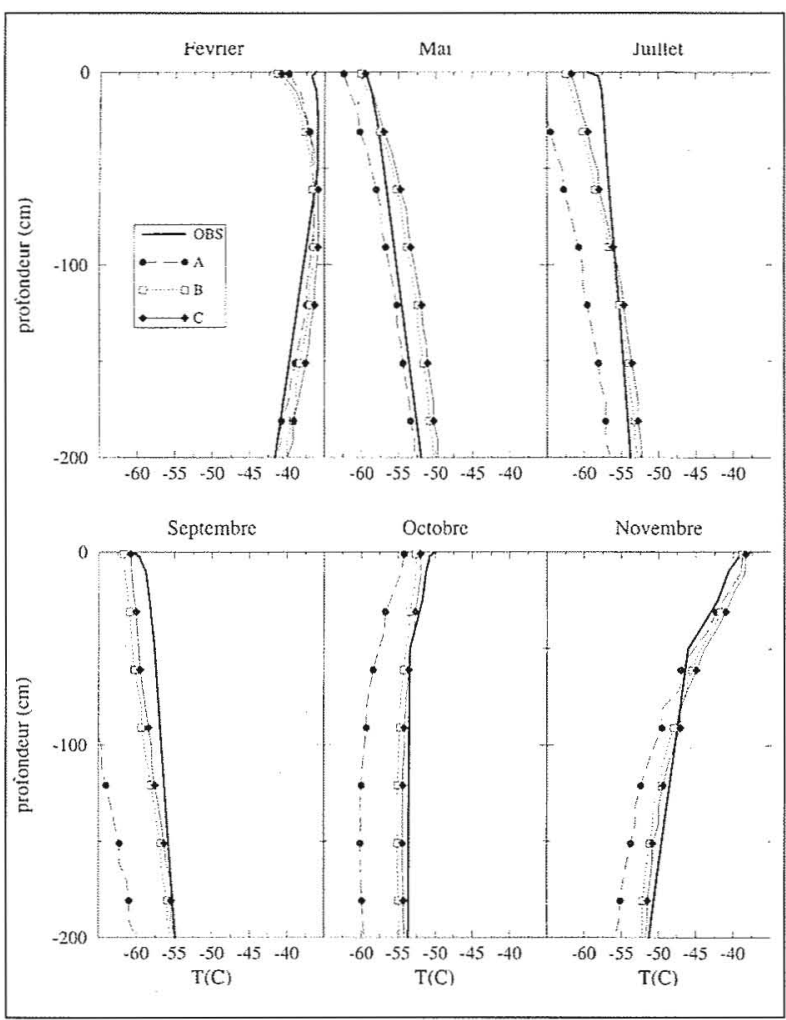

4. Profils de température moyenne mensuelle à l'intérieur du manteau neigeux. Simulation $\mathrm{A}$ : Forçage par Tsurf CEPMMT, B : cf A avec Tsurf AWS, C : cf B avec correction 10Wm-2 sur IR incident.

de profondeur. Trois simulations ont été réalisées et sont comparées aux observations rapportées par Dalrymple (1966) pour les années 1957-1958. Dans la première expérience (simulation $\mathrm{A}$ ), le modèle est entièrement forcé par les données d'archive du CEPMMT à Pole Sud. La simulation $\mathrm{B}$ est identique à la première, sauf pour la donnée de température de l'air qui est issue de l'observation par l'AWS de Pole Sud. Enfïn, la simulation $\mathrm{C}$ reproduit les conditions de la seconde, mais le bilan infrarouge est augmenté de 10 $\mathrm{Wm}-2$.

L'impact des températures trop basses du CEPMMT est visible pour les mois d'hiver, de juillet, septembre et octobre. Le forçage par la température de surface observée (AWS) entraîne une amélioration très nette des profils simulés pour tous les mois de l'année. Ceci prouve que CROCUS gère correctement le transfert de la chaleur à travers le manteau neigeux, mais que la sensibilité du modèle à une erreur de forçage par la température atmosphérique est importante. La simulation $\mathrm{C}$ ne montre qu'une légère amélioration pour les mois de nuit polaire durant lesquels la seule composante infrarouge du rayonnement subsiste. Thermiquement, le modèle de neige est assez peu sensible aux erreurs typiques de cette donnée de forçage.

\subsection{Densité, taille et forme des grains}

Nous nous intéressons maintenant aux autres caractéristiques physiques du manteau neigeux, la densité, la taille et la forme des grains (fig. 5). Les observations auxquelles sont confrontées le modèle ont été obtenues au cours des années 60 et sont rapportées par Bader [1] pour la densité et Gow [7] pour la taille des grains.

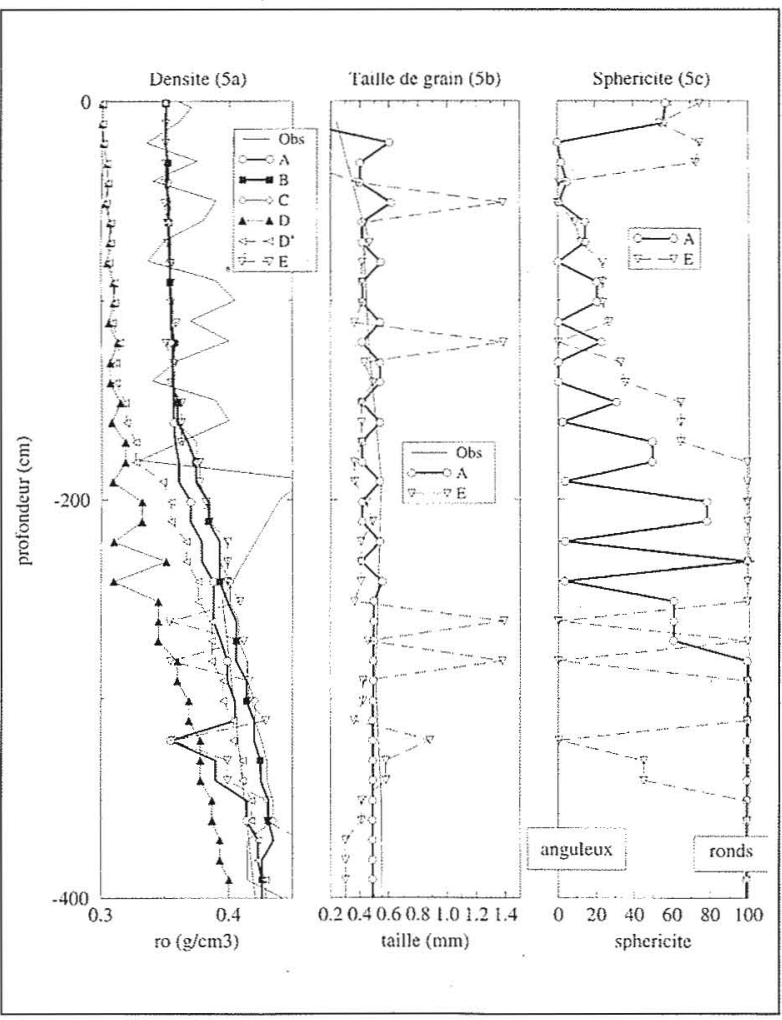

5. Profils de densité et de taille de grains à l'intérieur du manteau neigeux. Simulation A : Tsurf CEPMMT, B : Tsurf AWS, $\mathrm{C}$ : cf $\mathrm{B}+$ correction $10 \mathrm{Wm}^{-2}$ sur IR incident, $\mathrm{D}$ : cf $\mathrm{A}$ et densité en surface à $0.3 \mathrm{gcm}^{-3}, \mathrm{D}^{\prime}$ : cf $\mathrm{D}$ avec Tsurf AWS, E : ef B et perturbation sur l'accumulation.

Pour la densité (fig. 5a), les simulations A et B précédentes montrent une différence sensible uniquement à partir de $2 \mathrm{~m}$ de profondeur. Cela est dû au fait que la densification actuelle ne prend en compte que le tassement par le poids des couches supérieures et considère une viscosité compactive de la neige fonction de la densité et de la température dans le manteau. Ce tassement ne devient effectif dans le modèle qu'à partir d'environ $1.5 \mathrm{~m}$ de profondeur. Ceci ne correspond pas aux observations car dans les premiers mètres du manteau, on remarque une succession rapide de couches de densités différentes. Ce phénomène de densification rapide près de la surface est due au tassement par le vent et à la destruction des dendrites de la neige fraîchement déposée puis à l'agrégation des grains. Aucun de ces processus n'est actuellement simulé par le modèle.

Les résultats du modèle sont très sensibles à la densité initiale de la neige frâichement déposée à la surface. Dans CROCUS original, celle-ci est paramétrisée grâce à la formule de Pahaut [6]. Cette formule est valable pour la neige des régions tempérées, mais elle donne des densités beaucoup trop faibles pour les régions polaires.

En effet, dans ces régions froides et souvent ventées, il est habituel de mesurer des densités de surface s'échelonnant de

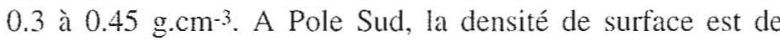
l'ordre de $0.35 \mathrm{~g}^{\prime} \mathrm{cm}^{-3}$ et nous avons modifié CROCUS de façon à imposer cette valeur à la place de la paramétrisation initiale. Les profils de densité montrent un bon accord avec les mesures (fig. 5a). Deux simulations supplémentaires (simulations D et D') ont été réalisées, dans lesquelles la

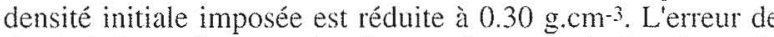
densité de surface ne s'atténue qu'à partir de 2 mètres de profondeur. A l'opposé, l'erreur de température de surface de l'archive CEPMMT ne se manifeste sur la densité simulée qu'à partir de 1.5 mètres (C'est en effet à cette profondeur 
que la compaction par le poids des couches supérieures, qui dépend de la température, devient importante). Clairement, une paramétrisation appropriée et précise de la densité de la neige de surface en fonction du vent et des autres paramètres météorologiques devra être développée avant que CROCUS ne puisse être considéré satisfaisant en régions polaires.

Pour la taille des grains (fig. $5 b$ ), la tendance générale est assez bien représentée si l'on considère la faible résolution verticale des observations. En fait, le modèle simule une succession régulière de couches de grains ronds et relativement petits et de grains anguleux et plus gros ( $f i g .5 c)$. Ces derniers correspondent à du givre de profondeur mais il semblerait que le modèle en produise trop fréquemment (données stratigraphiques non publiées de Jean-Robert Petit, communication personnelle). Effectivement, Perla et Ommaney [10] ont montré expérimentalement que les faibles densités $\left(<0.2 \mathrm{gcm}^{-3}\right)$ favorisaient la formation de givre de profondeur, mais qu'inversement les densités de l'ordre de celles rencontrées à Pole Sud supprimaient sa formation. Les lois paramétriques actuelles de CROCUS permettent cette formation même pour des fortes densités de neige, et ceci doit être corrigé.

La régularité, année après année, de la succession des couches provient de ce que l'accumulation imposée au modèle ne reproduit pas la variabilité interannuelle naturelle qui est importante. Une dernière simulation (simulation E) effectuée en variant arbitrairement l'accumulation d'une année sur l'autre (fig. 5) montre l'importance de ce paramètre sur la taille des grains. Effectivement, plus la précipitation est faible, plus les grains restent longtemps près de la surface, c'est-à-dire dans la zone de fort gradient de température, et plus ils ont tendance à grossir (jusqu'à plusieurs $\mathrm{mm}$ ) et à devenir anguleux [9].

\section{IV 娄 Conclusions}

L'extraction des données météorologiques des analyses du Centre européen de prévisions à météorologiques à moyen terme, a mis en évidence les incertitudes de ces données par rapport aux observations.

Les premiers tests de CROCUS montrent une forte sensibilité à la température de l'air imposée au modèle. Cette dépendance est importante dans la simulation des profils de températures à l'intérieur du manteau, et moindre pour ce qui est de la densité et de la taille des grains. L'incertitude sur le bilan de rayonnement infrarouge a un faible impact sur la simulation. L'accumulation par contre est un facteur important pour l'évolution de la taille des grains, comme l'ont montré ces tests.

Les valeurs initiales de densité et de taille de grains sont fonction des conditions météorologiques, vent et température de l'air. L'évolution du manteau neigeux dépend fortement de ces caractéristiques de la neige en surface. Les formulations de ces valeurs initiales doivent donc être adaptées aux conditions météorologiques polaires, afin de simuler correctement l'évolution du manteau.

Les résultats de cette étude sont dans l'ensemble très encourageants car même sans adaptation importante aux conditions polaires, CROCUS représente de façon correcte les grandes tendances de l'évolution du manteau neigeux.

Ce travail constitue la phase d'étude préliminaire du projet européen Polar Snow qui associe le Laboratoire de Glaciologie et Géophysique de l'Environnement, le Centre d'Etudes de la Neige, le British Antarctic Survey et la Universitat de Barcelona. Il sera poursuivi dans le cadre ce programme.

\section{Remerciements}

Le projet Polar Snow est financé par la Direction Générale XII de la Commission Européenne (contrat ENV4-CT950076).

\section{Références}

[1] BADER H. 1962 - Theory of densification of dry snow on high polar glaciers II. CRREL Research Report, 108.

[2] Brun E., Martin E., Simon V., Gendre C. and COLÉOU C. 1989 - An energy and mass model of snow cover suitable for operational avalanche forecasting. J. Glaciol., 35 (121), 333-342.

[3] Brun E., David P., Sudul M. and Brunot G. 1992 - A numerical model to simulate snow-cover stratigraphy for operational avalanche forecasting. J. Glaciol., 38 (128), 13-22.

[4] Carroll J.J. 1982 - Long term means and short term variability of the surface energy balance components at the south pole. J. Geophys. Res., 87 (C6), 4277-4286.

[5] Dalrymple, P. 1966 - A physical climatology of the Antarctic Plateau. In : Studies in Antarctic Meteorology, M.J. Rubin, editor, A.G.U. Antarctic Research Series 9: 195-231.

[6] Durand Y., Brun E., Merindol L., Guyomarc'H G., LESAFFrE B. and MARTIN E. 1993 - A meteorological estimation of relevant parameters for snow models. Ann. Glaciol., 18, 65-71.

[7] Gow A.J 1969 - On the rate of growth of grains and crystals in south polar firn. J. Glaciol., 8 (53), 241-252

[9] Nishimura H. and MaEno N. 1984 - Snow structure and depth hoar formation in Mizuho Plateau, Antarctica. Mem. Natl. Inst. Polar Res. Spec. Issue, 34, 137-146.

[10] Nishimura H. and Maeno N. 1985 - Studies on structures and physical properties of snow in Mizuho Plateall, Antarctica. Ann. Glaciol., 6, 105 107.

[11] Perla R. and Ommanney C.S.L. 1985 - Snow in strong or weak temperature gradients. Part 1. Experiments and qualitative observations. Cold Reg. Sci. Technol., 11 (1), 23-35. 